\title{
Epidermal growth factor receptor in adrenocortical tumors: analysis of gene sequence, protein expression and correlation with clinical outcome
}

\author{
Patrick Adam¹, Stefanie Hahner², Martina Hartmann ${ }^{3}$, Barbara Heinrich ${ }^{2}$, \\ Marcus Quinkler ${ }^{4}$, Holger S Willenberg ${ }^{5}$, Wolfgang Saeger ${ }^{6}$, Silviu Sbiera ${ }^{2}$, \\ Sebastian Schmull ${ }^{2}$, Hans-Ullrich Voelker ${ }^{3}$, Philipp Ströbel ${ }^{7}$, Bruno Allolio ${ }^{2}$ \\ and Martin Fassnacht ${ }^{2}$
}

\begin{abstract}
${ }^{1}$ Institute of Pathology, University of Tübingen, Tübingen, Germany; ${ }^{2}$ Department of Medicine I, Endocrine and Diabetes Unit, University Hospital, University of Würzburg, Würzburg, Germany; ${ }^{3}$ Institute of Pathology, University of Würzburg, Würzburg, Germany; ${ }^{4}$ Clinical Endocrinology, Charité Campus Mitte, Charité University Medicine Berlin, Berlin, Germany; ${ }^{5}$ Department of Endocrinology, Diabetes and Rheumatology, University Hospital Düsseldorf, Düsseldorf, Germany; ${ }^{6}$ Institute of Pathology, Marienkrankenhaus Hamburg, Hamburg, Germany and ${ }^{7}$ Institute of Pathology, Mannheim, University of Heidelberg, Heidelberg, Germany
\end{abstract}

\begin{abstract}
Adrenocortical carcinoma is a rare but highly malignant neoplasm with still limited treatment options. Epidermal growth factor receptor (EGFR) has been shown to be overexpressed in many solid tumors, but its expression in adrenocortical carcinoma has been studied only in a limited number of cases. Therefore, we analyzed the expression of EGFR in 169 adrenocortical carcinoma samples and compared it with 31 adrenocortical adenomas. Additionally, in 30 cases of adrenocortical carcinoma, exons 18-21 of the EGFR gene were cloned and sequenced. EGFR expression was found in 128 of 169 adrenocortical carcinoma samples $(76 \%)$, and in 60 of these samples $(=36 \%)$ strong membrane staining was detected. However, there was no significant correlation with clinical outcome. In addition, all 30 sequenced cases revealed unmutated EGFR genes. In contrast, only 1 out of 31 adrenocortical adenomas weakly expressed the EGFR (3\%). In summary, EGFR was overexpressed in more than three-quarters of adrenocortical carcinoma cases of this series. However, no mutations of the EGFR gene were found and EGFR expression was not of prognostic relevance. As EGFR is hardly expressed in adrenocortical adenomas, our results suggest that its expression in adrenocortical tumors indicates a malignant phenotype, which may be used in the differential diagnosis between adrenocortical adenomas and carcinomas.
\end{abstract}

Modern Pathology (2010) 23, 1596-1604; doi:10.1038/modpathol.2010.153; published online 6 August 2010

Keywords: adrenal cancer; adrenocortical adenoma; EGFR

Adrenal masses are among the most frequent tumors in humans. ${ }^{1}$ A vast majority of these tumors are benign adrenocortical adenomas and only a small subset are malignant adrenocortical carcinomas. ${ }^{2}$ Histologically, these tumor entities are discrimi-

Correspondence: Dr M Fassnacht, MD, Department of Medicine I, Endocrine and Diabetes Unit, University Hospital, University of Würzburg, Oberdürrbacher Str. 6, Würzburg 97080, Germany. E-mail: Fassnacht_m@medizin.uni-wuerzburg.de

Received 13 April 2010; revised 16 June 2010; accepted 17 June 2010; published online 6 August 2010 nated by scoring systems such as the Weiss score. ${ }^{3}$ Although tumors with a Weiss score of $\leq 1$ are clearly benign and tumors with a score of $\geq 4$ are without doubt malignant, there is often uncertainty in tumors with a Weiss score of 2 or 3 . However, up to now, no generally accepted marker has been established to determine malignancy in these tumors. $^{4,5}$ Adrenocortical carcinoma is a rare neoplasm with an estimated prevalence of 4-12 per million and a poor prognosis. ${ }^{6-9}$ Despite advances in the past decade, the precise pathogenesis is still poorly understood..$^{7,10-12}$ The clinical course of 
adrenocortical carcinoma is often rapidly progressing and apart from surgery for localized tumors no standardized therapy is established. ${ }^{8}$ The 5-year overall survival ranges between 25 and $50 \%$ in most series and in metastasized disease (stage IV) the median survival is only about 12-15 months. ${ }^{9}$

Epidermal growth factor receptor (EGFR/c-erbB1) was first characterized in epidermal carcinoma cells. ${ }^{13}$ Like the other growth factor receptors of the erbB family, EGFR consists of an extracellular domain, which binds the corresponding ligands, a transmembrane spanning domain and an intracellular tyrosine kinase domain. ${ }^{14}$ The encoding gene is located on chromosome 7 at 7 p12. Several ligands including epidermal growth factor, transforming growth factor- $\alpha$, amphiregulin, heregulin or betacellulin can activate the receptor. ${ }^{15}$ Ligand binding to the EGFR extracellular domain results in dimerization of the receptor complex, leading to activation of downstream signaling pathways and crossautophosphorylation of several other transmembrane receptors. ${ }^{16}$

EGFR has a pivotal role in tumorigenesis, ${ }^{17}$ with many human solid tumors overexpressing EGFR, including colon, ${ }^{18}$ breast and non-small cell lung cancer. ${ }^{19}$ For several tumor entities, a prognostic correlation between EGFR expression and clinical outcome has been demonstrated: Selvaggi et $a l^{20}$ found an overexpression of EGFR in non-small cell lung cancer in $>10 \%$ of tumor cells associated with a significantly shorter median survival time of 18 months when compared with 50 months in the control group. Also, the 5-year survival rates differed significantly with 31 versus $46 \%$ in the control group. Similarly, a large prospective study ${ }^{21}$ on 1029 patients with breast cancer without evidence of distant metastases indicated EGFR expression, in particular combined with the expression of the estrogen receptor, being a negative prognostic factor for disease-free and overall survival.

In recent years, EGFR has become a promising target for therapies in different tumor entities. For instance, the monoclonal anti-EGFR antibody cetuximab and the EGFR tyrosine kinase inhibitor erlotinib led (in monotherapy or in combination with cytotoxic drugs) to improved survival in patients with colorectal cancer, non-small cell lung cancer and advanced pancreatic cancer. ${ }^{22-24}$

Besides the expression of EGFR protein, there is evidence that the presence of activating mutations in the EGFR tyrosin kinase gene may confer exquisite sensitivity to EGFR inhibitors, but this remains a matter of debate. ${ }^{25-30} \mathrm{Up}$ to now, expression of EGFR has been studied only in four small series of adrenocortical tumors. Taken together, the results of these series show that EGFR was expressed in a vast majority of adrenocortical carcinoma samples $(n=127) .{ }^{31-34}$ However, none of these studies correlated EGFR expression with clinical outcome, nor did these studies analyze EGFR gene mutations. Therefore, we aimed at a comprehensive investigation of the EGFR system in adrenocortical carcinoma. In this study, we systematically analyzed EGFR expression in 169 adrenocortical carcinoma samples, 31 adrenal adenomas and 5 normal adrenal glands. In addition, we screened for mutations in the EGFR gene in 30 adrenocortical carcinoma samples and correlated the results with the clinical outcome of these patients.

\section{Materials and methods}

\section{Patients and Tissue}

Adrenal tumor tissue $(n=200)$ was collected from patients undergoing adrenalectomy for adrenocortical carcinoma $(n=169)$, aldosterone-producing adenoma $(n=12)$, cortisol-producing adenoma $(n=10)$ or endocrine-inactive adenoma $(n=9)$. The diagnosis was based on clinical, biochemical and morphological data. All histological diagnoses were confirmed by the reference pathologist of the German Adrenocortical Carcinoma Registry (WS). Clinical data were collected by the German Adrenocortical Carcinoma Registry (www.nebennieren karzinom.de) and included follow-up and survival data of adrenocortical carcinoma patients. Table 1 summarizes the clinical characteristics of the patients. All patients were Caucasians. Five normal adrenals derived from tumor nephrectomies served as controls.

The patients signed informed consent for collecting tissue and clinical data and the study was approved by the ethics committee of the University of Wuerzburg, Germany (approval number 93/02).

\section{Tissue Microarrays (TMAs)}

A total of 182 adrenocortical tumors (167 adrenocortical carcinomas and 15 adrenocortical adenomas) and five normal adrenal samples were assembled into three TMAs as described previously. ${ }^{35-37}$ Briefly, hematoxylin and eosin-stained sections of formalin-fixed and paraffin-embedded tissue blocks were evaluated to identify representative areas of well-preserved morphology. The corresponding area on the paraffin block was marked for tissue punching. The TMA was assembled using a commercially available manual tissue puncher/ arrayer (Beecher Instruments, Silver Spring, MD, USA) according to the manufacturer's instructions. Five cores with a diameter of $0.6 \mathrm{~mm}$ were punched from each tumor block and arrayed into a recipient paraffin block at a distance of $1 \mathrm{~mm}$ between each core. We chose to array five punch biopsies per case to minimize the number of cases not analyzable because of tissue loss and to increase concordance rates among different cores. Sections $(2 \mu \mathrm{m})$ cut on silanized slides were also used for conventional immunohistochemical stains. Before immunohistochemical staining, slides were dried 
Table 1 Patient characteristics

\begin{tabular}{|c|c|c|c|c|}
\hline $\begin{array}{l}\text { (a) } \\
\text { Type of tumor }\end{array}$ & $\begin{array}{l}\text { Normal adrenal } \\
\text { glands }\end{array}$ & $\begin{array}{c}\text { Aldosterone-producing } \\
\text { adrenocortical adenoma }\end{array}$ & $\begin{array}{c}\text { Cortisol-producing } \\
\text { adrenocortical adenoma }\end{array}$ & $\begin{array}{c}\text { Endocrine-inactive } \\
\text { adrenocortical adenoma }\end{array}$ \\
\hline$N$ & 5 & 12 & 10 & 9 \\
\hline Age & $59(44-83)$ & $45(31-69)$ & $53(35-73)$ & $61(43-76)$ \\
\hline $\operatorname{Sex}(M / F)$ & $1 / 4$ & $6 / 6$ & $1 / 9$ & $3 / 6$ \\
\hline Tumor size & - & $2(0.3-5)$ & $3(1.5-6)$ & $4(1-11)$ \\
\hline \multicolumn{5}{|c|}{ (b) Adrenocortical carcinomas } \\
\hline ENSAT stage & $I$ & $I I$ & III & $I V$ \\
\hline$N$ & 6 & 50 & 39 & 37 \\
\hline Age & $63(23-77)$ & $46(15-77)$ & $53(31-74)$ & $48(7-74)$ \\
\hline $\operatorname{Sex}(M / F)$ & $2 / 4$ & $21 / 29$ & $14 / 25$ & $10 / 27$ \\
\hline Tumor size & $4.5(3.0-5.0)$ & $11(6.0-28)$ & $10(5-20)$ & $13(6-25)$ \\
\hline \multicolumn{5}{|l|}{ Functional status ${ }^{a}$} \\
\hline $\begin{array}{l}\text { Glucocorticoids } \pm \text { other } \\
\text { steroids }\end{array}$ & 3 & 17 & 11 & 18 \\
\hline $\begin{array}{l}\text { Mineralocorticoids } \\
\text { only }\end{array}$ & 0 & 3 & 2 & 1 \\
\hline $\begin{array}{l}\text { Sex hormones/ } \\
\text { precursors only }\end{array}$ & 0 & 6 & 4 & 3 \\
\hline Endocrine inactive & 1 & 7 & 7 & 4 \\
\hline \multicolumn{5}{|l|}{ EGFR expression } \\
\hline 0 & 0 & 13 & 12 & 7 \\
\hline 1 & 1 & 9 & 8 & 6 \\
\hline 2 & 1 & 12 & 4 & 11 \\
\hline 3 & 4 & 16 & 15 & 13 \\
\hline
\end{tabular}

ENSAT, European Network for the Study of Adrenal Tumors staging system. ${ }^{47}$

${ }^{\mathrm{a}}$ In some patients no pre-surgical hormonal workup was performed.

Data presented as median and range.

Part (a) shows adrenocortical adenoma patients, and part (b) shows adrenocortical carcinoma patients in whom primary tumor was analyzed (in three patients, stage could not be determined).

at room temperature for 7 days in order to minimize tissue loss. For each block, hematoxylin and eosin-stained slides were cut to verify tumor cell content. A case was considered evaluable if at least two of the five cores contained tumor and if at least two cores were left for analysis after sectioning and the immunohistochemical staining procedure.

\section{Immunohistochemistry}

Immunohistochemical detection of EGFR expression was performed on standard sections of paraffinembedded tissue and the three TMAs using the EGFR pharmDx ${ }^{\text {тм }}$ test kit (DAKO, Hamburg, Germany) after proteinase K $(0.1 \%$ Proteinase K enzyme diluted in Tris-HCl buffer containing $0.015 \mathrm{~mol} / \mathrm{l}$ sodium azide) proteolytic digestion for $5 \mathrm{~min}$, according to the manufacturer's protocol. Endogeneous peroxidase was blocked by incubating the slides with $3 \%$ hydrogen peroxide for $5 \mathrm{~min}$. Subsequently, $100 \mu \mathrm{l}$ of the primary antibody, an $\mathrm{IgG}_{1}$ antibody against the extern domain of the EGFR molecule, was incubated for $30 \mathrm{~min}$ at room temperature. After rinsing the slides in fresh buffer
(Tris-buffered saline solution containing Tween 20, $\mathrm{pH}$ 7.6), the labeled polymer HRP (a dextran polymer conjugated with horseradish peroxidase and affinity-isolated goat-anti mouse immunoglobulins in Tris-HCl buffer) was applied. Slides were incubated for $30 \mathrm{~min}$ in a humid chamber. After rinsing the slides again in fresh buffer, the $\mathrm{DAB}+$ Substrate-Chromogen solution (5\%, 3,3'-diaminobenzidine in chromogen solution) was added and incubated for $10 \mathrm{~min}$. After washing in phosphatebuffered saline and $\mathrm{H}_{2} \mathrm{O}$, the slides were counterstained in hematoxylin.

Staining intensity was determined semiquantitatively using a four-grade $(0,1+, 2+$ and $3+)$ system. Cases, in which only $<10 \%$ of tumor cells were weakly stained, were judged as negative. In all samples with negative EGFR staining on the TMA, staining of the corresponding original tissue block was performed.

\section{DNA Extraction, PCR Procedures and Sequencing}

DNA from tumors and control tissues was isolated using the Trizol (guanidinium thiocyanate-phenolchloroform) extraction. For PCR amplification, 
Table 2 Sequences of primers used for the analysis of exons 18-21 of the epidermal growth factor receptor gene

$\begin{array}{ll}\text { Exon } 18 \mathrm{fw} & 5^{\prime} \text {-TCAGAGCCTGTGTTTCTACCAA-3' } \\ \text { Exon } 18 \mathrm{rev} & 5^{\prime} \text {-TGGTCTCACAGGACCACTGATT-3' } \\ \text { Exon } 19 \mathrm{fw} & 5^{\prime} \text {-AAATAATCAGTGTGATTCGTGGAG-3' } \\ \text { Exon } 19 \mathrm{rev} & 5^{\prime} \text {-GAGGCCAGTGCTGTCTCTAAGG-3' } \\ \text { Exon } 20 \mathrm{fw} & 5^{\prime} \text {-ACTTCACAGCCCTGCGTAAAC-3 } \\ \text { Exon } 20 \mathrm{rev} & 5^{\prime} \text {-ATGGGACAGGCACTGATTTGT-3' } \\ \text { Exon } 21 \mathrm{fw} & 5^{\prime} \text {-GCAGCGGGTTACATCTTCTTTC-3' } \\ \text { Exon } 21 \mathrm{rev} & 5^{\prime} \text {-CAGCTCTGGCTCACACTACCAG-3' }\end{array}$

fw, forward; rev, reverse.

$0.2 \mu \mathrm{g}$ DNA was amplified using Taq polymerase (Amersham, Freiburg, Germany) and sequence-specific primers (Table 2). The amplified PCR product was loaded onto a preparative agarose gel, excised, and extracted using the Jetsorb gel extraction kit (Jetsorb, Bad Oeynhausen, Germany). In all, 3-5 $\mu$ l of the product was used for direct sequencing of both strands using the ABI sequencing mix (Applied Biosystems, Weiterstadt, Germany). After denaturation, the samples were subjected to electrophoresis using an ABI 373A sequencer (Applied Biosystems) and analyzed using the GenScan software (Applied Biosystems). Resulting sequences were aligned to each other and to a published wild-type sequence (GenBank accession number AF288738).

\section{Statistical Analysis}

The Kruskal-Wallis test and $\chi^{2}$ test were used to assess the significance of associations between categorical data, as appropriate. The mean values of continuous data were compared using Student's $t$-test or ANOVA with post hoc analysis carried out using Fisher's PLSD test. Survival probabilities were estimated using the univariate Kaplan-Meier method, and survival curves were compared using the log-rank test. Correlations between EGFR expression and other morphological/immunohistochemical factors that have been shown to be predictive of tumor behavior were analyzed using the two-tailed Pearson analysis. Statistical differences with $P$-values $<0.05$ were considered significant. Analyses were performed using the statistical software package SPSS, version 13.0 (SPSS, Chicago, IL, USA).

\section{Results}

Of the 161 adrenocortical carcinomas analyzable in the TMA staining (6 cases were not evaluable), 96 showed a specific membranous positivity for EGFR. With respect to the well-known heterogeneous distribution of EGFR within the tumor tissue, ${ }^{38}$ all cases with no specific staining on the TMA and additionally 8 sections from samples not included in the TMAs were stained in sections from the corresponding original tissue paraffin blocks. Therefore, a total of 169 adrenocortical carcinoma samples could be analyzed (Table 3). EGFR staining was
Table 3 Epidermal growth factor receptor expression in adrenal tissue samples

\begin{tabular}{lrrrr}
\hline EGFR expression score & $0+$ & $1+$ & $2+$ & $3+$ \\
\hline (a) & & & & \\
ACC primary tumor $(n=129)$ & 53 & 20 & 18 & 38 \\
ACC local recurrence $(n=18)$ & 7 & 2 & 1 & 8 \\
ACC distant metastases $(n=14)$ & 5 & 6 & 1 & 2 \\
Normal adrenal gland $(n=5)$ & 5 & 0 & 0 & 0 \\
Aldosterone-producing ACA $(n=5)$ & 4 & 1 & 0 & 0 \\
Cortisol-producing ACA $(n=5)$ & 5 & 0 & 0 & 0 \\
Endocrine inactive ACA $(n=4)$ & 4 & 0 & 0 & 0 \\
& & & & \\
(b) & & & & \\
ACC primary tumor $(n=135)$ & 33 & 24 & 30 & 48 \\
ACC local recurrence $(n=19)$ & 4 & 1 & 4 & 10 \\
ACC distant metastases $(n=15)$ & 4 & 6 & 3 & 2 \\
Normal adrenal gland $(n=5)$ & 5 & 0 & 0 & 0 \\
Aldosterone-producing ACA $(n=12)$ & 11 & 1 & 0 & 0 \\
Cortisol-producing ACA $(n=10)$ & 10 & 0 & 0 & 0 \\
Endocrine-inactive ACA $(n=9)$ & 9 & 0 & 0 & 0
\end{tabular}

Epidermal growth factor receptor expression score: 0, no; 1+, weak; $2+$, moderate; and $3+$, strong membrane staining.

Part (a) shows adrenal tissue samples analyzable in the tissue microarrays, and part (b) shows all adrenal tissue samples after staining the original tissue specimens in all samples that were epidermal growth factor receptor negative on the tissue microarray+additional 6 adrenocortical carcinomas and 18 adrenocortical adenoma samples.

strong in 60 cases $(36 \%)$ including 27 samples, in which $>90 \%$ of all tumor cells showed strong staining intensity. In addition, EGFR immunoreactivity was moderate (score $2+$ ) in 36 cases $(21 \%)$ and weak (score $1+)$ in 32 cases $(19 \%)$, whereas in 41 cases $(24 \%)$ no specific staining could be detected (score 0) (Figure 1).

In contrast, EGFR staining was negative in all normal adrenal tissues $(n=5)$ and in 32 of 33 adrenal adenomas. Only one aldosterone-producing adenoma showed a weak (grade 1) expression of EGFR. Therefore, positive EGFR staining was of diagnostic value in discriminating adrenocortical adenoma from carcinoma (sensitivity $76 \%$, specificity $97 \%$, positive predictive value $99 \%$ and negative predictive value $44 \%$ ).

In adrenocortical carcinoma, the EGFR expression was similar in tumor specimens derived from surgery of local recurrence or metastases (for details see Table 3). In addition, there was no correlation between EGFR staining and sex, age, tumor size and endocrine secretion and there was no association between EGFR staining intensity and tumor stage (Table 1). In 18 patients, we had the opportunity to analyze tumor samples from consecutive surgeries (surgery at the time of primary diagnosis and surgery for recurrent disease). In seven of these cases the intensity of EGFR staining was unchanged, in ten it was reduced in the specimen of the second surgery and in one case it was increased from score $2+$ to score $3+$, further suggesting that EGFR expression in adrenocortical carcinoma is not a sign of a more aggressive behavior. In agreement with this finding, univariate and multivariate analyses did not reveal 

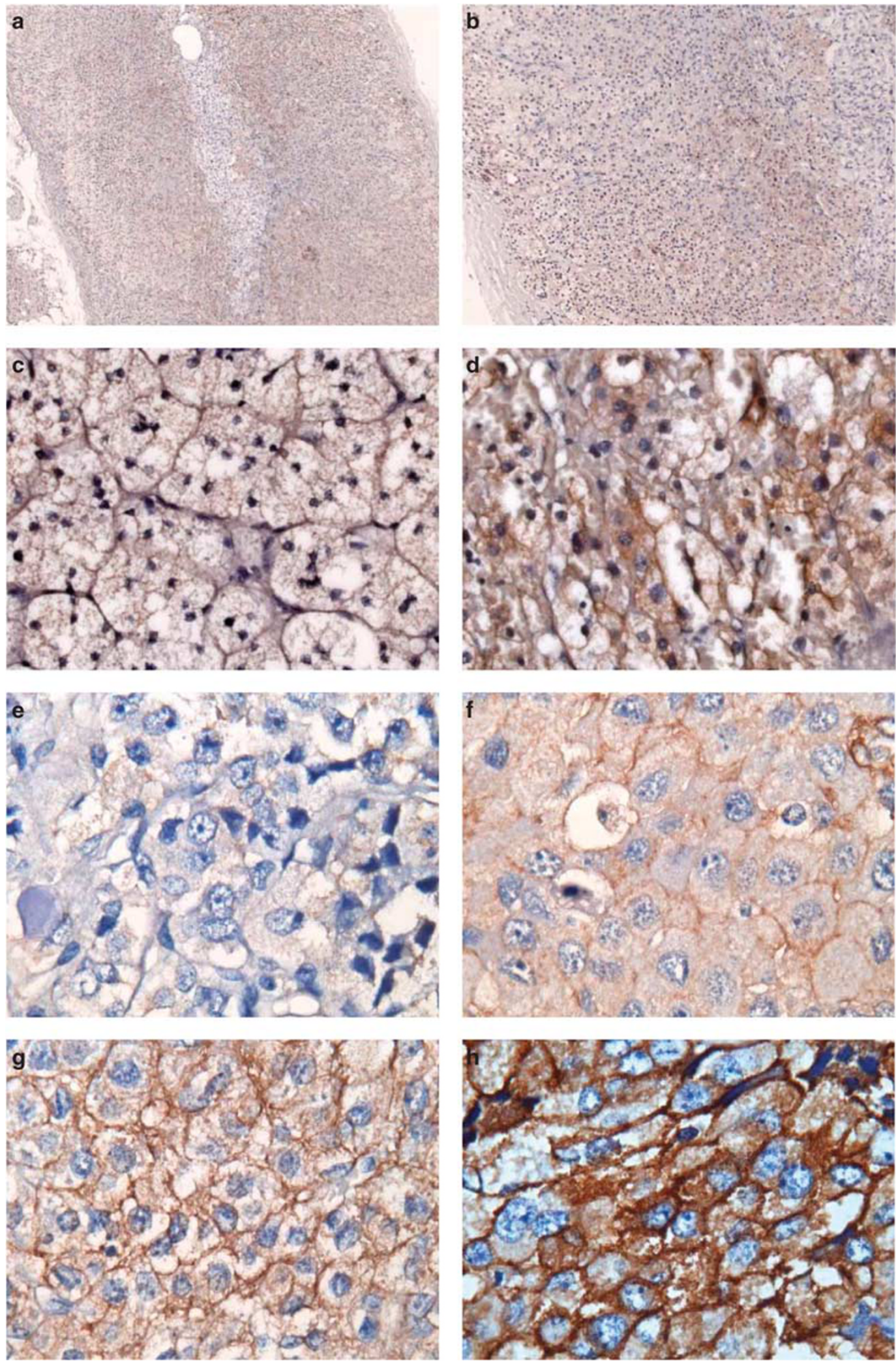

Figure 1 Immunohistochemical detection of epidermal growth factor receptor (EGFR) in adrenocortical tissues (immunoperoxidase). (a, b) Normal adrenal gland did not show a specific membrane staining for EGFR (a, original magnification $\times 50$; b, original magnification $\times 100$ ). (c) Adrenocortical adenomas in their majority did not express EGFR (original magnification $\times 400$ ). (d) Only a single adrenocortical adenoma showed a weak membrane positivity in $>10 \%$ of tumor cells (original magnification $\times 400$ ). (e-h) Adrenocortical carcinoma samples: (e) no (score 0), (f) weak (score 1), (g) moderate (score 2) and (h) strong membrane EGFR expression (score 3) (all original magnification $\times 400$ ). 


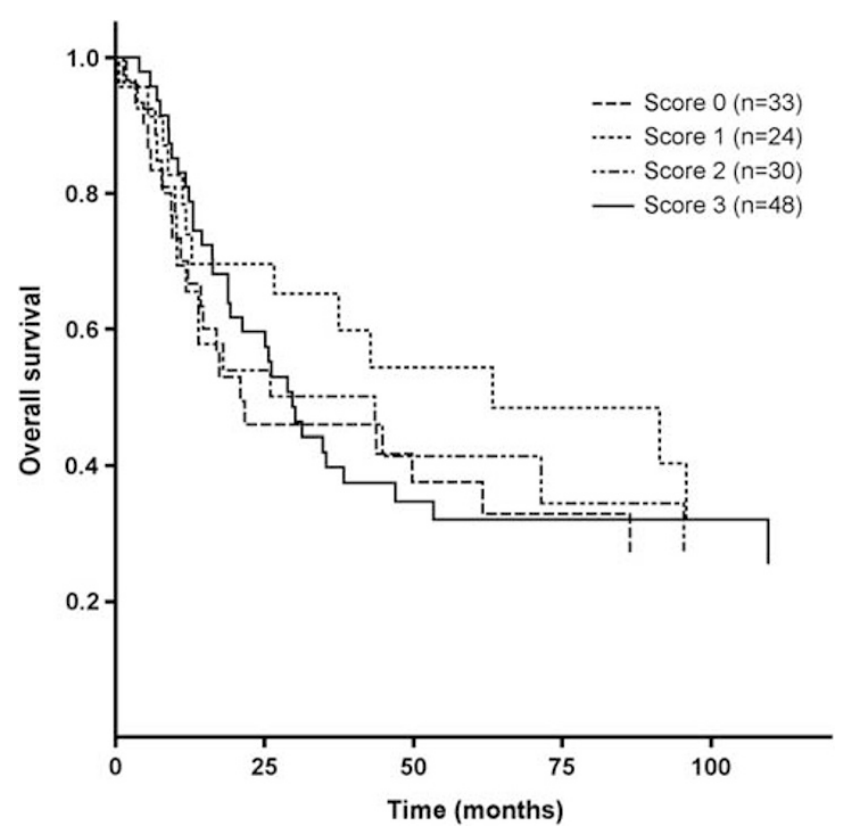

Figure 2 Kaplan-Meier survival analysis comparing adrenocortical carcinoma patients with different epidermal growth factor receptor (EGFR) staining intensity of their primary tumor. Only patients with detailed follow-up information are included in this analysis.

any correlation of EGFR staining intensity and overall survival in 133 patients in whom the primary tumor was analyzed (Figure 2).

Furthermore, we have correlated the expression of the EGFR with morphological features and immunohistochemical markers that have been shown to be predictive of tumor behavior. ${ }^{31,35,38}$ However, this analysis yielded only a weak correlation with the Weiss score $(r=0.178, P=0.049)$, whereas the expression of Ki67 $(r=0.137, P=0.234)$ and GLUT1 $(r=0.089, P=0.343)$ did not correlate significantly with the EGFR score.

Additionally, in order to determine possible mutations in the EGFR leading to constitutional activation of the EGFR tyrosine kinase activity, tumor DNA covering exons 18-21 of the EGFR gene was sequenced in 30 cases. Analysis of all sequences revealed $100 \%$ identity to a published EGFR reference sequence (AF288738). Genotype distribution among three known single-nucleotide polymorphisms (SNPs), $154737 \mathrm{G}>\mathrm{T}$ (G719C) in exon 18, 162093G $>A$ (Q787Q) in exon 19 (dbSNP_rs1050171) and 172480C $>\mathrm{T}$ (R836R) in exon 21 (dbSNP_rs17290559), was similar to published frequencies within the European population (frequencies obtained from http://www.ncbi.nlm. nih.gov/SNP).

\section{Discussion}

In this study we confirm in a large series that the EGFR is expressed in $>75 \%$ of adrenocortical carcinomas. However, there was no correlation of
EGFR expression with clinical outcome nor did we find mutations in the EGFR gene. EGFR staining was negative in all normal adrenal tissues and in 32 of 33 adrenal adenomas, suggesting EGFR expression as an indicator of a malignant phenotype. Up to now, only few immunohistochemical markers have been established for distinguishing adrenocortical carcinomas from adenomas. ${ }^{39-43}$ However, most of these markers were investigated only in small series and none of them has reached general acceptance. In this context, staining for EGFR might be clinically of great interest: although negative EGFR staining obviously does not rule out adrenocortical carcinoma, our findings indicate that a tumor with moderate or strong EGFR expression is almost certainly malignant. EGFR staining is routinely used in a variety of tumor entities. ${ }^{20,38}$ Provided our findings are confirmed in an independent cohort, it will also be easy to use EGFR staining in adrenocortical tumors of uncertain malignant potential.

Sequencing analysis of exons 18-21 of the EGFR gene yielded no mutations, and genotype distribution among three known SNPs in exons 18, 20 and 21 was similar to published frequencies within the European population. This lack of mutations does not preclude a role of the EGFR in the biology of adrenocortical carcinoma, as mutations that render the receptor ligand independent are not the only mechanisms by which EGFR can contribute to tumor growth. Alternative pathomechanisms include gene amplification and overexpression of a non-mutated receptor with increased sensitivity to ligand binding. ${ }^{44}$

Overexpression of EGFR in adrenocortical carcinoma has already been described in four small studies. $^{31-34}$ However, our series included more samples than all four series taken together; in addition, some of these studies did not differentiate cytoplasmatic from membranous staining. Nowadays, only the latter would be judged as a specific staining. Moreover, this is the first time that correlation studies between EGFR expression and survival were performed, because detailed clinical follow-up data derived from the German adrenocortical carcinoma registry were available. However, in contrast to studies in some other tumor entities, ${ }^{20,21}$ we could not find a significant association between EGFR expression and overall survival of patients. Therefore, our study does not provide evidence that a high EGFR expression is related to an aggressive behavior of these tumors. Accordingly, we could not find an association between EGFR expression and metastatic tumor spread as was described by Kamio et $a .^{32}$ The finding that tumor tissue derived from surgery for local recurrence or metastases did not show a stronger EGFR expression in comparison to the primary tumor, points in the same direction. Although EGFR expression is an indicator of malignancy in adrenocortical tumors, strong EGFR expression apparently does not provide the tumor with a significant advantage to grow or to metastasize. 
Evidence that the EGFR pathway is not a key oncogenic signaling pathway in adrenocortical carcinomas is also supported by clinical experience with drugs targeting EGFR. In 10 patients with advanced adrenocortical carcinoma, the combination of the EGFR inhibitor erlotinib with gemcitabine did not lead to any objective tumor response, and preliminary similar results were reported for gefitinib. ${ }^{45,46}$

During our work-up all cases with no specific staining for EGFR on the TMAs were stained in sections from the corresponding original tissue blocks. Although a majority of cases $(n=41 ; 63 \%)$ remained negative, focal expression of EGFR was observed in the other cases (weak $n=7$, moderate $n=11$ and strong $n=6$ ), demonstrating the known heterogeneous expression of EGFR that has also been described for other tumor entities. This observation clearly demonstrates that TMA analysis in adrenocortical carcinomas should be performed preferentially if not exclusively for proteins with homogeneous expression, as otherwise laborious re-stainings of presumably negative results have to be performed. As we did not repeat staining in all samples, we cannot exclude that we missed some samples with strong focal staining intensity that might have led to a change in score. However, re-categorizing few samples would probably not have changed the impact on survival, because even within the cohort of patients with very strong EGFR staining in $>90 \%$ of all tumor cells of the primary tumor $(n=22)$, survival was very heterogeneous (median: 22 months; range 4-134 months). Furthermore, categorizing all samples exclusively based on their TMA score did not significantly affect outcome analysis.

In an attempt to elucidate a possible correlation of EGFR expression with parameters that have been shown to be predictive of tumor behavior, ${ }^{31,35,38}$ we found only a weak correlation with the Weiss score, whereas the expression of Ki67 and GLUT1 did not correlate significantly with EGFR expression.

In conclusion, our results confirm that immunohistochemically detectable EGFR is overexpressed in more than three-quarters of adrenocortical carcinoma samples. In contrast, membranous EGFR staining is virtually not present in adrenocortical adenomas, suggesting that EGFR expression in adrenal tumors indicates a malignant phenotype and therefore might be of diagnostic relevance.

Mutations of the EGFR gene were not found in our series, suggesting that a constitutively activated EGFR cascade is not a major oncogenic pathway in adrenocortical carcinoma. In addition, EGFR expression does not correlate with clinical outcome, indicating that it is not a promising therapeutic target in adrenocortical carcinoma.

\section{Acknowledgements}

This study was supported by grants of the Deutsche Krebshilfe (no. 106080 to BA and MF and grant no.
107111 to MF), the German Ministry of Research BMBF (no. 01KG0501 to BA and MF) and the German Research Foundation (DFG; FA 466/3-1 to MF). This study was part of the German adrenal network GANIMED (German Adrenal Network Improving Treatment and Medical Education). The following pathologists provided tumor material from two or more patients for the tissue array: Gerhard Seitz (Klinikum Bamberg), Harald Stein, Manfred Dietel, (Charite University Berlin), Gerhard Mall (Klinikum Darmstadt), Helmut Erich Gabbert (University of Duesseldorf), Werner Schmid (University of Essen), Steffen Hauptmann (Martin-Luther University of Halle), Peter Schirrmacher (University of Heidelberg), Alfred C Feller (University of Luebeck), C James Kirkpatrick (University of Mainz), Roland Moll (University of Marburg), Cyrus Tschahargane (Lukaskrankenhaus, Neuss), Rainer Horst Krech (Klinikum Osnabrueck), Ferdinand Hofstaedter (University of Regensburg), Andrea M Gassel (Leopoldina Hospital Schweinfurt). The following hospitals/clinicians contributed clinical data from three or more patients to the German Adrenocortical Cancer Registry: Wolfgang Oelkers (University Hospital Charite Berlin), Michael Morcos (University Hospital Heidelberg), Peter Langer (University Hospital of Marburg), Christian Fottner (University Hospital Mainz), Michael Brauckhoff (University Hospital Halle), Felix Beuschlein (University of Freiburg/Munich), Horst L Fehm (University Hospital Lübeck), Dagmar Führer (University Hospital Leipzig), Stephan Petersenn (University Hospital Essen, now in Hamburg). We appreciate the support of Uwe Maeder (Tumor Center, University Hospital Wuerzburg) in establishing the German ACC registry database and are thankful to Michaela Haaf for documentation.

\section{Disclosure/conflict of interest}

The authors declare no conflict of interest.

\section{References}

1 Grumbach MM, Biller BM, Braunstein GD, et al. Management of the clinically inapparent adrenal mass ('incidentaloma'). Ann Intern Med 2003;138:424-429.

2 Mansmann G, Lau J, Balk E, et al. The clinically inapparent adrenal mass: update in diagnosis and management. Endocr Rev 2004;25:309-340.

3 Weiss LM, Medeiros LJ, Vickery Jr AL. Pathologic features of prognostic significance in adrenocortical carcinoma. Am J Surg Pathol 1989;13:202-206.

4 Saeger W. Histopathological classification of adrenal tumours. Eur J Clin Invest 2000;30(Suppl 3):58-62.

5 Sasano H, Suzuki T, Moriya T. Recent advances in histopathology and immunohistochemistry of adrenocortical carcinoma. Endocr Pathol 2006;17:345-354.

6 Wajchenberg BL, Albergaria Pereira MA, Medonca BB, et al. Adrenocortical carcinoma: clinical and laboratory observations. Cancer 2000;88:711-736. 
7 Libe R, Fratticci A, Bertherat J. Adrenocortical cancer: pathophysiology and clinical management. Endocr Relat Cancer 2007;14:13-28.

8 Schteingart DE, Doherty GM, Gauger PG, et al. Management of patients with adrenal cancer: recommendations of an international consensus conference. Endocr Relat Cancer 2005;12:667-680.

9 Allolio B, Fassnacht M. Clinical review: adrenocortical carcinoma: clinical update. J Clin Endocrinol Metab 2006;91:2027-2037.

10 Kirschner LS. Signaling pathways in adrenocortical cancer. Ann NY Acad Sci 2002;968:222-239.

11 Sidhu S, Sywak M, Robinson B, et al. Adrenocortical cancer: recent clinical and molecular advances. Curr Opin Oncol 2004;16:13-18.

12 Libe R, Bertherat J. Molecular genetics of adrenocortical tumours, from familial to sporadic diseases. Eur J Endocrinol 2005;153:477-487.

13 Krupp MN, Connolly DT, Lane MD. Synthesis, turnover, and down-regulation of epidermal growth factor receptors in human A431 epidermoid carcinoma cells and skin fibroblasts. J Biol Chem 1982;257: 11489-11496.

14 Bargmann CI, Hung MC, Weinberg RA. The neu oncogene encodes an epidermal growth factor receptor-related protein. Nature 1986;319:226-230.

15 de Boer WI, Hau CM, van Schadewijk A, et al. Expression of epidermal growth factors and their receptors in the bronchial epithelium of subjects with chronic obstructive pulmonary disease. Am J Clin Pathol 2006;125:184-192.

16 Alroy I, Yarden Y. The ErbB signaling network in embryogenesis and oncogenesis: signal diversification through combinatorial ligand-receptor interactions. FEBS Lett 1997;410:83-86.

17 Yarden Y. The EGFR family and its ligands in human cancer. Signalling mechanisms and therapeutic opportunities. Eur J Cancer 2001;37(Suppl 4):S3-S8.

18 Nygren P, Sorbye H, Osterlund P, et al. Targeted drugs in metastatic colorectal cancer with special emphasis on guidelines for the use of bevacizumab and cetuximab: an Acta Oncologica expert report. Acta Oncol 2005;44:203-217.

19 Shelton JG, Steelman LS, Abrams SL, et al. The epidermal growth factor receptor gene family as a target for therapeutic intervention in numerous cancers: what's genetics got to do with it? Expert Opin Ther Targets 2005;9:1009-1030.

20 Selvaggi G, Novello S, Torri V, et al. Epidermal growth factor receptor overexpression correlates with a poor prognosis in completely resected non-small-cell lung cancer. Ann Oncol 2004;15:28-32.

21 Tsutsui S, Ohno S, Murakami S, et al. Prognostic value of epidermal growth factor receptor (EGFR) and its relationship to the estrogen receptor status in 1029 patients with breast cancer. Breast Cancer Res Treat 2002;71:67-75.

22 Cunningham D, Humblet Y, Siena S, et al. Cetuximab monotherapy and cetuximab plus irinotecan in irinotecan-refractory metastatic colorectal cancer. N Engl J Med 2004;351:337-345.

23 Shepherd FA, Rodrigues Pereira J, Ciuleanu T, et al. Erlotinib in previously treated non-small-cell lung cancer. N Engl J Med 2005;353:123-132.

24 Moore MJ, Goldstein D, Hamm J, et al. Erlotinib plus gemcitabine compared with gemcitabine alone in patients with advanced pancreatic cancer: a phase III trial of the National Cancer Institute of Canada Clinical Trials Group. J Clin Oncol 2007;25:1960-1966.

25 Lynch TJ, Bell DW, Sordella R, et al. Activating mutations in the epidermal growth factor receptor underlying responsiveness of non-small-cell lung cancer to gefitinib. N Engl J Med 2004;350:2129-2139.

26 Paez JG, Janne PA, Lee JC, et al. EGFR mutations in lung cancer: correlation with clinical response to gefitinib therapy. Science 2004;304:1497-1500.

27 Tsao MS, Sakurada A, Cutz JC, et al. Erlotinib in lung cancer-molecular and clinical predictors of outcome. N Engl J Med 2005;353:133-144.

28 Marchetti A, Felicioni L, Buttitta F. Assessing EGFR mutations. N Engl J Med 2006;354:526-528; author reply 526-528.

29 Chan SK, Gullick WJ, Hill ME. Mutations of the epidermal growth factor receptor in non-small cell lung cancer-search and destroy. Eur J Cancer 2006;42:17-23.

30 Sequist LV, Joshi VA, Janne PA, et al. Response to treatment and survival of patients with non-small cell lung cancer undergoing somatic EGFR mutation testing. Oncologist 2007;12:90-98.

31 Edgren M, Eriksson B, Wilander E, et al. Biological characteristics of adrenocortical carcinoma: a study of p53, IGF, EGF-r, Ki-67 and PCNA in 17 adrenocortical carcinomas. Anticancer Res 1997;17:1303-1309.

32 Kamio T, Shigematsu K, Sou H, et al. Immunohistochemical expression of epidermal growth factor receptors in human adrenocortical carcinoma. Hum Pathol 1990;21:277-282.

33 Sasano H, Suzuki T, Shizawa S, et al. Transforming growth factor alpha, epidermal growth factor, and epidermal growth factor receptor expression in normal and diseased human adrenal cortex by immunohistochemistry and in situ hybridization. Mod Pathol 1994; 7:741-746.

34 Nakamura M, Miki Y, Akahira J, et al. An analysis of potential surrogate markers of target-specific therapy in archival materials of adrenocortical carcinoma. Endocr Pathol 2009;20:17-23.

35 Fenske W, Volker HU, Adam P, et al. Glucose transporter GLUT1 expression is an stage-independent predictor of clinical outcome in adrenocortical carcinoma. Endocr Relat Cancer 2009;16:919-928.

36 Ronchi CL, Sbiera S, Kraus L, et al. Expression of excision repair cross complementing group 1 and prognosis in adrenocortical carcinoma patients treated with platinum-based chemotherapy. Endocr Relat Cancer 2009;16:907-918.

37 Weismann D, Briese J, Niemann J, et al. Osteopontin stimulates invasion of NCI-h295 cells but is not associated with survival in adrenocortical carcinoma. J Pathol 2009;218:232-240.

38 Ioachim E, Kamina S, Athanassiadou S, et al. The prognostic significance of epidermal growth factor receptor (EGFR), C-erbB-2, Ki-67 and PCNA expression in breast cancer. Anticancer Res 1996;16:3141-3147.

39 Volante M, Sperone P, Bollito E, et al. Matrix metalloproteinase type 2 expression in malignant adrenocortical tumors: diagnostic and prognostic significance in a series of 50 adrenocortical carcinomas. Mod Pathol 2006;19:1563-1569.

40 Wachenfeld C, Beuschlein F, Zwermann O, et al. Discerning malignancy in adrenocortical tumors: are molecular markers useful? Eur J Endocrinol 2001;145:335-341.

41 Babinska A, Sworczak K, Wisniewski P, et al. The role of immunohistochemistry in histopathological diagnostics 
of clinically 'silent' incidentally detected adrenal masses. Exp Clin Endocrinol Diabetes 2008;116:246-251.

42 Schmitt A, Saremaslani P, Schmid S, et al. IGFII and MIB1 immunohistochemistry is helpful for the differentiation of benign from malignant adrenocortical tumours. Histopathology 2006;49:298-307.

43 Marx C, Wolkersdorfer GW, Brown JW, et al. MHC class II expression-a new tool to assess dignity in adrenocortical tumours. J Clin Endocrinol Metab 1996;81: 4488-4491.

44 Kuan CT, Wikstrand CJ, Bigner DD. EGF mutant receptor vIII as a molecular target in cancer therapy. Endocr Relat Cancer 2001;8:83-96.
45 Quinkler M, Hahner S, Wortmann S, et al. Treatment of advanced adrenocortical carcinoma with erlotinib plus gemcitabine. J Clin Endocrinol Metab 2008;93: 2057-2062.

46 Fassnacht M, Kreissl MC, Weismann D, et al. New targets and therapeutic approaches for endocrine malignancies. Pharmacol Ther 2009;123: 117-141.

47 Fassnacht $\mathrm{M}$, Johanssen S, Quinkler $\mathrm{M}$, et al. Limited prognostic value of the 2004 UICC staging classification for adrenocortical carcinoma - proposal for a revised TNM classification. Cancer 2009; 115:243-250. 